\title{
Tooth size in individuals with congenitally missing teeth: a study of Japanese males
}

\author{
Hiroyuki YAMADA $^{1 *}$, Shintaro KONDO ${ }^{1}$, Hajime HANAMURA ${ }^{1}$, Grant C. TownSEND ${ }^{2}$ \\ ${ }^{1}$ Department of Anatomy, School of Dentistry, Aichi-Gakuin University, Nagoya 464-8650, Japan \\ ${ }^{2}$ School of Dentistry, The University of Adelaide, Adelaide, South Australia 5005, Australia
}

Received 6 July 2009; accepted 31 August 2009

\begin{abstract}
There is evidence of a relationship between tooth size and tooth agenesis in human populations, with a tendency for reduced tooth size in individuals with missing teeth. The aim of this study was to analyze the size of remaining teeth (mesiodistal crown diameters) and their variability in individuals with varying degrees of severity of congenitally missing teeth. Tooth crown diameters were recorded from 100 dental plaster casts of Japanese males. Subjects were divided into three agenesis groups: group A had one missing tooth per individual, group B had two missing teeth per individual, and group $\mathrm{C}$ had three or more missing teeth per individual. For comparison, tooth size data were used from a previously studied sample of Japanese males who did not have any congenitally missing teeth. Group A displayed the largest mesiodistal crown size dimensions for all maxillary and mandibular teeth, followed by group B, and then group C. In comparison with the reference data, when only one or two teeth were missing, the remaining teeth tended to be larger, but when there were three or more missing teeth, the remaining teeth tended to be significantly smaller throughout the dentition. In terms of tooth size variability, dental dimensions in the agenesis groups were generally more variable compared with those in the reference sample. This tendency was most pronounced in the group with the greatest number of missing teeth. Although there was a tendency for the size of the remaining teeth to be more reduced as the number of missing teeth increased, tooth size in individuals with only one or two teeth missing was generally larger than in a control group with all 32 permanent teeth, suggesting that the relationship between tooth size and dental agenesis may be more complicated than previously thought, perhaps due to local compensatory interactions affecting the size of teeth.
\end{abstract}

Key words: agenesis, mesiodistal crown diameters, tooth reduction, high variability, humans

\section{Introduction}

It has been reported that tooth size has become smaller and tooth number has been reduced due to a general reduction in the size of the jaws during the course of human evolution (Keith, 1924; Bolk, 1916; Hellman, 1936). With regard to third molar agenesis, Brothwell et al. (1963) noted that congenitally missing third molars were not frequent in the Upper Paleolithic but that they were more common during the Neolithic era in Europe. Third molar agenesis has also been reported in Southeast Asia, and frequencies are quite high in the northern part of this area. Indeed, the prevalence rate of over $30 \%$ is greater than reports for any other region of the world (Brothwell et al., 1963). On the other hand, frequencies of dental agenesis, excluding third molars, have been reported to be lie between about 2 and $7 \%$ for other human populations (Werther and Rothenberg, 1939;

* Correspondence to: Hiroyuki Yamada, Department of Anatomy, School of Dentistry, Aichi-Gakuin University, 1-100 Kusumoto-cho, Chikusa-ku, Nagoya 464-8650, Japan.

E-mail: ymd_20hiro4@ybb.ne.jp

Published online 20 October 2009

in J-STAGE (www.jstage.jst.go.jp) DOI: 10.1537/ase.090706
Zimmerman, 1967; Muller et al., 1970; Hermal et al., 1971; Thompson and Popovich, 1974; Yamada and Hanamura, 1993). Congenitally missing teeth frequently involve the distal teeth within each tooth class. These distally situated teeth generally develop later than the mesial ones, and their later development seems to predispose them to congenital absence that is significant in phylogenic terms (Fujita, 1958; Ozaki, 1960).

When congenitally missing teeth are reported in the dentition, the remaining teeth tend to show dental reduction. Garn et al. (1961a, b; 1963a, b) and Garn and Lewis (1970) reported in the dentitions of American Whites that if the third molars were congenitally missing, the remaining teeth were more likely to show reduction in crown size, morphology, and developmental timing compared with individuals with a full complement of teeth. Furthermore, crown size was generally more reduced in a multiple agenesis group (lacking third molars and other teeth) than in individuals with simple third molar agenesis (Garn and Lewis, 1970). In contrast, Sofaer et al. (1971a) and Sofaer (1973) reported in schoolchildren in Hawaii that when the maxillary lateral incisor was congenitally absent on one side, the adjacent central incisor was larger in size than its counterpart, suggesting a possible compensatory local interaction affecting the size of 
adjacent teeth. A similar phenomenon of an increase in tooth size of Japanese dentition has also been reported in individuals with agenesis of four third molars (Asakura, 1975; Yamada et al., 2005).

This study aims to determine whether there is any trend evident in the size of the remaining teeth in the second dentition of Japanese males who display varying degrees of severity of missing teeth. Three groups of individuals with different degrees of severity of dental agenesis are compared with each other and also with a reference group with all 32 permanent teeth present. We hypothesize that the remaining teeth will show increased tooth size compared with the controls when the number of congenitally missing teeth is small, but that tooth size will be progressively reduced as the number of missing teeth increases.

\section{Materials and Methods}

The study material used consisted of the dental plaster casts made from alginate impressions of the dentitions of 100 Japanese males, aged from 10 to 30 years, and the majority of subjects were born between 1949 and 1970. The models are stored at Aichi-Gakuin University, Nagoya, Japan. The congenital absence of teeth was confirmed radiographically for all affected individuals. Only individuals with permanent dentitions were included in the study. Medical histories in each case showed no experience of previous tooth loss due to trauma, dental caries, periodontal disease, or orthodontic extractions. Only subjects without systemic illnesses or genetic medical conditions were included.

The subjects with agenesis of teeth were divided into three groups and were compared with a reference sample of Japanese males, for whom data had already been collected by one of the authors (Yamada et al., 2005). The first group (group A) consisted of individuals with only one missing tooth per person (59 males), the second group (group B) had two missing teeth per person (21 males), and the third group (group C) had three or more missing teeth per person (20 males), excluding third molars. The individuals in the reference or control group all had complete permanent dentitions, with 32 permanent teeth confirmed radiographically, including third molars. They came from the Tokai region of Japan (Yamada et al., 2005).

The permanent teeth from the central incisor to the second premolar of both jaws were measured on the dental casts by the first author alone (H.Y.) following the method of Fujita (1949) using sliding calipers (manufactured by Mitsutoyo Company, Japan) to an accuracy of $0.05 \mathrm{~mm}$. The averages of right- and left-sided teeth were obtained but if a tooth on one side could not be measured due to congenital absence, dental caries, presence of restorations, or an abnormality in form, the corresponding tooth on the other side was accepted.

Measurement errors were analyzed by a procedure in which double determination measurements were made on separate occasions for 50 subjects selected at random. Differences between first and second determinations at intervals of 6 months or more were analyzed by computing the standard deviation of a single determination (Dahlberg, 1940).

Comparisons were made based on basic descriptive statistics, and the percentage differences between the average me- siodistal crown diameters of the agenesis groups and those of the reference group were also calculated using the following formula: [(agenesis group mean-reference group mean)/reference group mean] $\times 100$. In addition, as a representation of overall crown size in a study sample, the total of average tooth size (TATS), which was devised by H.Y., was calculated by summarizing the averages of the mesiodistal crown diameters in the maxilla and the mandible.

Descriptive statistics, including mean values and standard deviations, were calculated for all tooth size variables using JMP statistical software version 5.1 for Windows (SAS Institute, 2000) on a personal computer. Differences between the agenesis tooth groups and the reference group were analyzed for mean values with the Tukey-Kramer HSD test (Tukey, 1953; Kramer, 1956) and for variances with the $F$-test.

\section{Results}

There was no indication of systematic measurement errors between first and second determinations, based on paired $t$-tests. Mean differences between first and second determinations ranged from -0.00 to $0.01 \mathrm{~mm}$. Measurement errors, indicated by the Dahlberg (1940) statistic, ranged in value from $0.06 \mathrm{~mm}$ for the lateral incisor to $0.10 \mathrm{~mm}$ for the canine in both jaws. When error variance was expressed as a percentage of total observed variance, error percentages for mesiodistal diameters ranged from 0.67 to $1.78 \%$. Overall, errors of the measurement were small for all variables, and unlikely to bias the results of this study.

The total number of missing teeth was 186; therefore there were on average 1.9 missing teeth per person. The most frequent tooth to be missing was the mandibular second premolar $(23.7 \%)$, followed by the maxillary second premolar (21.5\%), maxillary lateral incisor (17.2\%) and mandibular first incisor (14.0\%) (Table 1).

Based on the TATS values calculated from central incisor to second premolar for both jaws, group A $(73.7 \mathrm{~mm})$ showed largest tooth size, then group B $(72.9 \mathrm{~mm})$, followed by group $C(69.5 \mathrm{~mm})$. The reference group showed an intermediate tooth size of $72.1 \mathrm{~mm}$ (Table 2).

The results of comparisons of tooth size between the three agenesis groups (A, B and C), using Tukey-Kramer HSD tests are shown in Table 3, with the significance levels indicated. Group A had largest teeth and group $\mathrm{C}$ smallest in all

Table 1. Frequencies of agenesis for different tooth types

\begin{tabular}{lrrc}
\hline \multicolumn{1}{c}{ Tooth } & $\begin{array}{c}\text { No. of teeth } \\
\text { (subjects) }\end{array}$ & $\begin{array}{c}\text { Percentage of teeth } \\
\text { (\%) }\end{array}$ \\
\hline Mandibular P2 & 44 & $(29)$ & 23.7 \\
Maxillary P2 & 40 & $(28)$ & 21.5 \\
Maxillary I2 & 32 & $(25)$ & 17.2 \\
Mandibular I1 & 26 & $(21)$ & 14.0 \\
Mandibular I2 & 21 & $(17)$ & 11.3 \\
Maxillary P1 & 13 & $(9)$ & 7.0 \\
Maxillary C & 8 & $(6)$ & 4.3 \\
Mandibular P1 & 1 & $(1)$ & 0.5 \\
Mandibular C & 1 & $(1)$ & 0.5 \\
Maxillary I1 & 0 & $(0)$ & 0.0 \\
\hline Total & $186(137)$ & 100.0 \\
\hline
\end{tabular}


Table 2. TATS ${ }^{1}$ values in agenesis and reference groups (in $\mathrm{mm}$ )

\begin{tabular}{lc}
\hline & Male \\
\hline Group A & 73.7 \\
Group B & 72.9 \\
Group C & 69.5 \\
Total (A, B, C) & 72.8 \\
Reference & 72.1 \\
\hline
\end{tabular}

${ }^{1}$ TATS: Sum of the averages for mesiodistal diameters from central incisor to second premolar of both jaws

dental measurements, with the exception of the mandibular central incisor where group B was slightly larger than group A. No significant differences in mean values between groups $\mathrm{A}$ and $\mathrm{B}$ were found in either the maxilla or the mandible. In contrast, group A showed significantly larger tooth size than group C, except for the mandibular second premolar. Group $B$ also showed significantly larger incisors than those of group C, but no significant differences were found in the canine and premolars in both jaws. Table 3 also shows comparisons of three agenesis groups with the reference data. All teeth in group A were larger than in the control or reference group. Significant differences were found for maxillary central incisors $(P<0.01)$ and mandibular premolars $\left(\mathrm{P}_{1}\right.$ : $\left.P<0.01 ; \mathrm{P}_{2}: P<0.05\right)$. Group $\mathrm{B}$ also tended to display larger tooth size than the reference group, but there were no significant differences. However, group $\mathrm{C}$ displayed definitely smaller tooth dimensions throughout the dentition than the reference group. The differences were statistically significant for lateral incisors $\left(\mathrm{I}^{2}: P<0.01 ; \mathrm{I}_{2}: P<0.05\right)$ of both jaws (Figure 1 and Figure 2).

The results for tooth size variability in the agenesis groups compared with the reference group, as indicated by variance ratio, are provided in Table 4 . In the maxilla, canine teeth of groups $\mathrm{B}$ and $\mathrm{C}$ were significantly more variable in tooth size variability, and in the mandible the following teeth were significantly more variable: the lateral incisor in group A; lateral incisor and canine in group B; and central incisor, canine, and second premolar in group $\mathrm{C}$.

\section{Discussion}

Tooth size is thought to follow a polygenic mode of inheritance like many other quantitative physical characters, and it appears that dental agenesis occurs when developing tooth germs fall below a certain critical size (Falconer, 1981). Brook (1984) and Brook et al. (2002) have proposed a multifactorial model to relate tooth size and number that is based on an underlying continuous distribution of tooth size, with thresholds determining hypodontia and supernumeraries. Brook hypothesized that hypodontia is linked to small tooth size, whereas supernumerary teeth are associated with larger tooth size.

Congenitally missing teeth in the dentition have been showed to be associated with size reduction of the remaining teeth (Garn et al., 1963a). Garn et al. (1963a, b) noted in American whites that the greater the number of missing teeth, the smaller in size the remaining teeth tended to be. Hanihara (1970) described in the Pima Indians that individuals with reduced or conical maxillary lateral incisors had smaller teeth than those without altered lateral incisors. Reduction in tooth number was associated with reduction in crown size, morphology, and developmental timing of the remaining teeth, and furthermore, crown size was generally reduced in individuals with multiple agenesis (Garn and Lewis, 1970). A direct relationship was postulated between tooth agenesis and decreased tooth size, with nearly $70 \%$ of permanent teeth being significantly smaller in size in an agenesis group (up to six teeth missing) (Baum and Cohen, 1971a). Lavelle et al. (1970) also reported in British whites that mesiodistal crown diameters were smaller on average in individuals with third molar agenesis compared with those with complete dentitions. More recently, Brook et al. (2002, 2009) reported that patients with hypodontia tended to have smaller remaining teeth than a control group and that this difference was statistically significant.

However, in the case of a study using the mouse dentition, Grüneberg $(1963,1965)$ found that if the first molar of the mouse, which was the largest in the tooth row normally, was reduced to become the smallest, then the second and third molars tended to grow even larger than in a normal mouse.

Table 3. Comparison of mean values for mesiodistal crown diameters between agenesis and reference groups

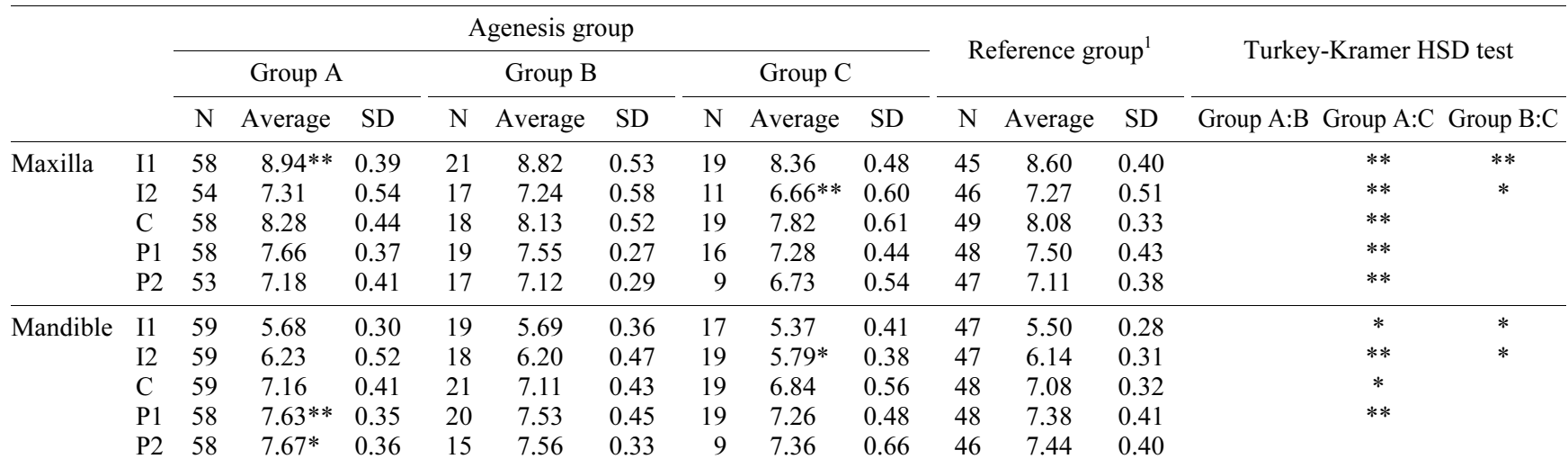

${ }^{1}$ Data from Yamada et al. (2005)

$* P<0.05,{ }^{* *} P<0.01$ among three agenesis and reference groups (Tukey-Kramer HSD test) 


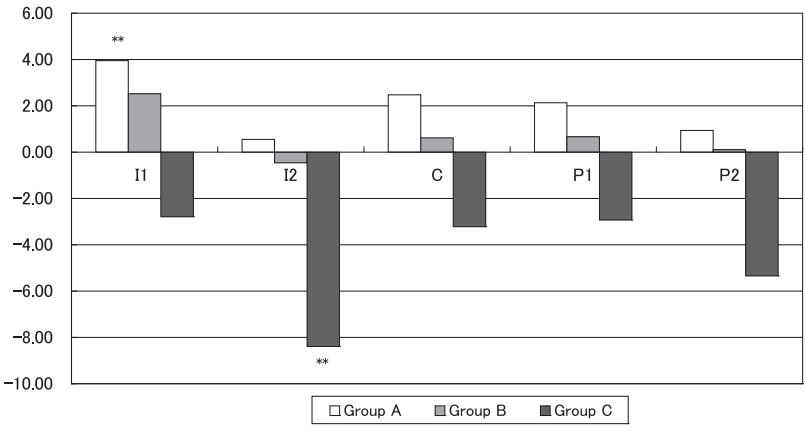

Figure 1. Percentage differences of average maxillary mesiodistal crown diameters in agenesis groups compared with the reference group ${ }^{1}(\%)$

${ }^{1}$ Data from Yamada et al. (2005)

* $P<0.05,{ }^{* *} P<0.01$ between three agenesis groups and reference group (Tukey-Kramer HSD test)

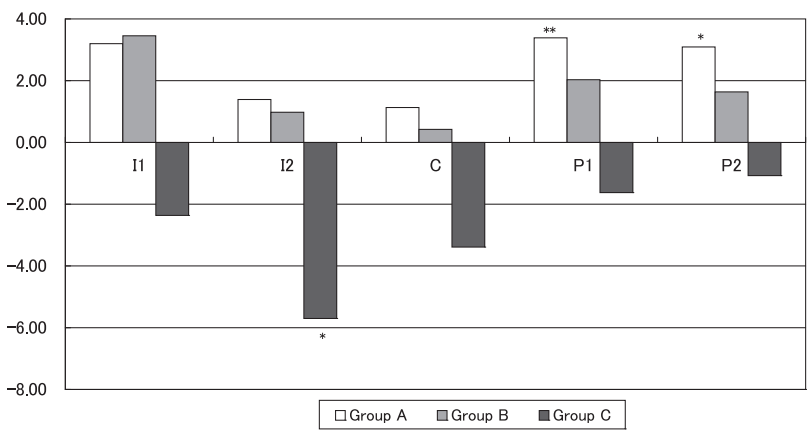

Figure 2. Percentage differences of average mandibular mesiodistal crown diameters in agenesis groups compared with the reference group ${ }^{1}(\%)$

${ }^{1}$ Data from Yamada et al. (2005)

$* P<0.05, * * P<0.01$ between three agenesis groups and reference group (Tukey-Kramer HSD test)

Sofaer et al. (1971a, b) also reported in schoolchildren in Hawaii that the adjacent central incisor of humans tended to be larger when the lateral incisor alongside it was congenitally missing. Furthermore, it has been noted that certain teeth were significantly larger in an agenesis group with four third molars missing than in a group with full complements of 32 permanent teeth, for both females (Asakura, 1975) and males (Yamada et al., 2005). Therefore, it would seem that tooth agenesis is associated in some general way with dental reduction, but the crown size of the remaining teeth is not always reduced - indeed, in some cases it may be increased.

In total, 186 teeth were congenitally absent in the 100 individuals examined in the present study. The most frequent tooth to be missing, excluding third molars, was the mandibular second premolar in males (23.7\%), a finding that is consistent with previous studies (Dolder, 1936; Terasaki and Siota, 1954; Grahnen, 1956; Brown, 1957; Volk, 1963; Rose, 1966; Horowitz, 1966; Ringqvist and Thilander, 1969; Haavikko, 1971; Hermal et al., 1971; Hirukawa et al., 1999). In contrast, some researchers have reported that the maxillary lateral incisor is the most frequently congenitally missing tooth (Werther and Rothenberg, 1939; Okamoto et al., 1951; Zimmerman, 1967; Baum and Cohen, 1971a, b).
Table 4. Tooth size variability in crown size of permanent teeth of three agenesis groups

Variability expressed as F ratio (variance of tooth agenesis group to variance of reference group ${ }^{1}$ )

\begin{tabular}{llllll} 
Maxilla & UI1 & UI2 & UC & UP1 & UP2 \\
\hline A group & 0.94 & 1.13 & 1.80 & 0.73 & 1.14 \\
B group & 1.76 & 1.30 & $2.48^{* *}$ & 0.38 & 0.58 \\
C group & 1.44 & 1.38 & $3.42^{* *}$ & 1.05 & 2.02 \\
\hline & & & & & \\
\hline Mandible & LI1 & LI2 & LC & LP1 & LP2 \\
\hline A group & 1.16 & $2.81^{* *}$ & 1.64 & 0.73 & 0.81 \\
B group & 1.65 & $2.30^{*}$ & $1.81^{*}$ & 1.20 & 0.68 \\
C group & $2.12^{*}$ & 1.52 & $3.06^{* *}$ & 1.35 & $2.72^{*}$ \\
\hline
\end{tabular}

${ }^{1}$ Data from Yamada et al. (2005)

* $P<0.05,{ }^{* *} P<0.01$ between three agenesis groups

A critical issue to consider in comparing tooth size data between different study samples is the validity of the mesiodistal crown size measurements. Great care was taken to ensure that the caliper beaks were placed in the correct positions on all teeth to record their maximum mesiodistal diameters. This ensured that dimensions were not overestimated in teeth that did not have contact with adjacent teeth on the mesial and distal. In addition, where adjacent teeth were present, the caliper tips were placed interproximally to ensure that the maximum diameter of each tooth was recorded. As stated earlier, all measurements were made by the same researcher (H.Y.) to ensure that no interobserver errors were introduced. In addition, replicability studies confirmed that errors of the method were generally small and unlikely to bias results.

Among three agenesis groups, group A had the largest teeth, followed by group B and then group C. When tooth agenesis was severe, with many teeth missing, the remaining teeth tended to be reduced considerably in crown size. Yamazaki (1960) has reported that in Japanese the mesiodistal crown diameters of remaining teeth tended to be smaller in individuals with more than eight missing teeth. McKeown et al. (2002) also described in Caucasians that there was a trend for tooth dimensions of all teeth to be smaller in individuals with the severe agenesis (six or more congenitally missing teeth).

In oligodontia and tooth agenesis cases, PAX9 mutations have been described in families with inherited oligodontia (where many teeth are congenitally missing), characteristically involving permanent molars (Peters et al., 1998). In addition to permanent molars, other congenitally missing teeth have also been noted in the premolar, canine, and incisor regions (Lammi et al., 2003). In these cases, tooth size was also reduced. MSX1 mutations may also cause familial tooth agenesis (Lidral and Reising, 2002), with knockout mice for these genes also lacking teeth and displaying teeth with arrested development at the bud stage (Satokata and Maas, 1994; Peters et al., 1998). Thus, it would appear that in cases of oligodontia, the condition is strongly affected by genetic factors and the whole dentition tends to be reduced in size.

The TATS value for the reference group in the present study, calculated from central incisor to second premolar, was $72.1 \mathrm{~mm}$. This value is almost the same as those of 
Sakai (1957), whose data were collected from individuals in the Nagano and Yamanashi prefectures of Japan, at $72.0 \mathrm{~mm}$; Hanihara (1977), whose data were from Tokyo, at $71.9 \mathrm{~mm}$; and Yamada (1977), whose data came from the Tokai region, in central Japan, at $72.0 \mathrm{~mm}$. All of the analyses in the studies of Sakai (1957), Hanihara (1977), and Yamada (1977) did not take account of the third molars.

Interestingly, the reference group showed intermediate tooth size based on the TATS value when compared with the three agenesis groups. Intriguingly, group A displayed significantly larger mesiodistal diameters than the reference group. A similar trend was noted for group B, although no significant differences were found. In contrast, group $\mathrm{C}$ showed smaller tooth size and significant differences were found for lateral incisors of both jaws. The result for group C is consistent with those of Garn and Lewis (1970), Baum and Cohen (1971a), Brook et al. (2002), and Brook et al. (2009). Their data were obtained from Caucasians of European ancestry. That is, the more congenitally missing teeth, the smaller the mesiodistal crown diameters of the remaining teeth.

However, the results based on group A and group B provide further evidence that the size of remaining teeth may actually be increased in individuals with a small number of missing teeth. Yamada et al. (2005) reported in Japanese males that an agenesis group with four missing third molars had significantly larger first and second molars in both jaws (TATS $=72.9 \mathrm{~mm}$ ) than a reference group with all 32 permanent teeth (TATS $=72.1 \mathrm{~mm})$. Sofaer $(1973)$ proposed a model of compensatory interaction in maxillary incisors and claimed that compensation in size was most likely to be apparent when there was absence of one of the members of the class. It is suggested that when there are only one or two congenitally missing teeth, the remaining teeth may be increased in size in their mesiodistal direction, possibly due to compensatory interaction between adjacent teeth, but when there are more than three missing teeth, excluding third molars, the remaining teeth will tend to be significantly smaller, reflecting a multifactorial pattern of inheritance that affects the dentition overall. Given that the findings of the present study contrast with those of Brook et al. (2009), it is possible that the balance between compensatory factors acting locally within the dentition and more general genetic influences operating on the whole dentition may differ between ethnic groups. Further studies in different ethnic groups are needed to confirm whether true population differences do indeed exist. Nevertheless, as far as the Japanese dentition is concerned, our hypothesis that the remaining teeth tend to be larger when the number of congenitally missing teeth is small, but reduced in size when agenesis is severe, appears valid.

In relation to tooth size variability, the agenesis groups were generally more variable than the reference group. This tendency was most apparent when greater numbers of teeth were missing. Baum and Cohen $(1971 \mathrm{a}, \mathrm{b})$ noted that 6 out of 14 mesiodistal measurements were significantly more variable in males with tooth agenesis when compared with a control group. They reported that highest variation in tooth size variability was noted for the canine and first molar in the agenesis sample. Brook et al. (2009) also mentioned that the agenesis group in their study was more variable than the control in terms of tooth size, as did Yamada et al. (2005). It is possible that individuals with dental agenesis display increased developmental instability (Shapiro, 1975; Townsend, $1983 \mathrm{a}, \mathrm{b})$, and that this is reflected in greater variability in size of their remaining teeth.

Among the three agenesis groups in the present study, canines in groups $\mathrm{B}$ and $\mathrm{C}$ showed greatest variability. Mandibular incisors and second premolars were also variable in the agenesis groups. The fact that canine teeth were more variable in the agenesis groups is an apparent contradiction to the widely accepted notion that these teeth are stable key teeth in terms of Butler's field theory (Butler, 1939). Osborne et al. (1958) reported that canines showed lower genetic variability than other teeth, so it is interesting that they displayed relatively high phenotypic variation in the agenesis groups in our study. Further studies are needed to explore this finding.

This study did not consider the third molar in the agenesis groups. Tooth size of other remaining teeth in groups with congenitally missing teeth, including third molar agenesis, will be explored and compared with controls with 32 permanent teeth in the future.

\section{Conclusion}

Influences of congenital missing teeth on the size of the remaining teeth were explored. When compared with reference data for individuals with a full complement of 32 permanent teeth, individuals with one or two missing teeth generally had larger remaining teeth. When three or more teeth were congenitally missing, the remaining teeth tended to be smaller. These apparently contradictory findings may reflect an interplay between local compensatory effects on teeth adjacent to missing teeth and more general genetic influences operating on the dentition overall. The remaining teeth of individuals with missing teeth tended to be more variable in size than those of individuals with full dentitions, perhaps reflecting increased developmental instability.

\section{Acknowledgments}

We would like to express our grateful thanks to the students of Aichi-Gakuin University for their kind consent to collect dental plaster casts of their teeth. In addition, we wish to particularly thank Dr Shinichi Muroi for gathering materials of individuals with congenitally missing tooth. This study was supported in part by Grants-in-Aid for Scientific Survey No. 61041072 and No. 02041045 from the Ministry of Education, Culture, Sports, Science and Technology of Japan.

\section{References}

Asakura M. (1975) Relationships of size and form of the remaining teeth to third molar agenesis. Aichi-Gakuin Dental Science, 13: 270-302 (in Japanese with English summary).

Baum B.J. and Cohen M.M. (1971a) Studies on agenesis in the permanent dentition. American Journal of Physical Anthropology, 35: 125-128.

Baum B.J. and Cohen M.M. (1971b) Agenesis and tooth size in the 
permanent dentition. Angle Orthodontist, 41: 100-102.

Bolk L. (1916) Problems of human dentition. American Journal of Anatomy, 19: 91-148.

Brook A.H. (1984) A unifying aetiological explanation for anomalies of human tooth number and size. Archives of Oral Biology, 29: 373-378.

Brook A.H., Elcock C., Al-Sharood M.H., Mckeown H.F., Khalaf K., and Smith R.N. (2002) Further studies of a model for the etiology anomalies of tooth number and size in humans. Connective Tissue Research, 43: 289-295.

Brook A.H., Griffin R.C., Smith R.N., Townsend G.C., Kaur G., Davis G.R., and Fearne J. (2009) Tooth size patterns in patients with hypodontia and supernumerary teeth. Archives of Oral Biology, 54 (Supplement 1): S63-S70.

Brothwell D.R., Carbonell V.M., and Goose D.H. (1963) Congenital absence of teeth in human populations. Brothwell D.R. (ed.), Dental Anthropology. Pergamon Press, London, pp. 179-190.

Brown R.V. (1957) The pattern and frequency of congenital absence of teeth. Iowa Dental Journal, 43: 60-61.

Butler P.M. (1939) Studies of the mammalian dentition: differentiation of the post canine dentition. Proceedings of Zoological Society in London, B109: 1-36.

Dahlberg G. (1940) Statistical Methods for Medical and Biological Students. George Allen and Unwin, London, pp. 127-132.

Dolder E. (1936) Zahn-Unterzahl. Schweizerische Monatsschr Zahnheilkd, 46: 663-701.

Falconer D.S. (1981) Introduction to Quantitative Genetics, 2nd edn. Longman, New York:, pp. 340.

Fujita T. (1949) On the standard of the measurement of teeth. Journal of Anthropological Society of Nippon, 61: 27-32 (in Japanese with German summary).

Fujita T. (1958) Abnormality of tooth number in the human dentition. Journal of the Japanese Stomatological Society, 25: 97106 (in Japanese).

Garn S.M. and Lewis A.B. (1970) The gradient and pattern of crownsize reduction in simple hypodontia. Angle Orthodontist, 40: 51-58.

Garn S.M., Lewis A.B., and Vicinus J.H. (1961a) Third molar agenesis and reduction in the number of other teeth. Journal of Dental Research, 24: 717.

Garn S.M., Lewis A.B., and Bonne B. (1961b) Third molar polymorphism and the timing of tooth formation. Nature, 4806: 989.

Garn S.M., Lewis A.B., and Kerewsky R.S. (1963a) Third molar agenesis and size reduction of the remaining teeth. Nature, 200: 488-489.

Garn S.M., Lewis A.B., and Vicinus J.H. (1963b) Third molar polymorphism and its significance to dental genetics. Journal of Dental Research, 42 (Supplement): 1344-1363.

Grahnen H. (1956) Hypodontia in the permanent dentition. A clinical and genetical investigation. Odontological Review 7, Supplement 3, Thesis.

Grüneberg H. (1963) The Pathlogy of Development: A Inherited Skeletal Disorders in Animals. Blackwell Science, Oxford.

Grüneberg H. (1965) Genes and genotypes affecting the teeth of the mouse. Journal of Embryology and Experimental Morphology, 14: 137-159.

Haavikko K. (1971) Hypodontia of permanent teeth. An orthopantomographic study. Suomen Hammaslaakariseuran Toimituksia, 67: 219-225.

Hanihara K. (1970) Upper lateral incisor variability and the size of the remaining teeth. Journal of the Anthropological Society of Nippon, 78: 316-323.

Hanihara K. (1977) Distances between Australian aborigines and certain other populations based on dental measurements. Journal of Human Evolution, 6: 403-418.

Hellman M. (1936) Our third molar teeth, their eruption, presence and absence. Dental Cosmos, 78: 750.

Hermal J., Hermal G., and Ulmansky M. (1971) Dental abnormali- ties. Israel Journal of Dental Medicine, 20: 1-4.

Hirukawa K., Iwata R., Kurosawa M., Kondo K., and Goto S. (1999) Statistical investigation about the prevalence of congenitally missing permanent teeth. Orthodontics Waves, 58: 49-56 (in Japanese with English summary).

Horowitz J.M. (1966) Aplasia and malocclusion: a survey and appraisal. American Journal of Orthodontics, 52: 440-453.

Keith A. (1924) The Antiquity of Man. Williams and Norgate, London.

Kramer C.Y. (1956) Extension of multiple range tests to group means with unequal numbers of replications. Biometrics, 12: 309-310.

Lammi L., Halonen K., Pirinen S., Thesleff I., Arte S., and Nieminen P. (2003) A missense mutation in PAX9 in a family with distinct phenotype of oligodontia. European Journal of Human Genetics, 11: 866-871.

Lavelle C.L.B., Ashton E.H., and Flinn R.M. (1970) Cusp pattern, tooth size and third molar agenesis in the human mandibular dentition. Archives Oral Biology, 15: 227-237.

Lidral A.C. and Reising B.C. (2002) The role of MSX1 in human tooth agenesis. Journal of Dental Research, 81: 274-278.

McKeown H.F., Robinson D.L., Elcock C., Al-Sharood M., and Brook A.H. (2002) Tooth dimensions in hypodontia patients, their unaffected relatives and a control group measured by a new image analysis system. European Journal of Orthodontics, 24: 131-141.

Muller T.P., Hill I.N., Petersen A.C., and De George F.V. (1970) A survey of congenitally missing permanent teeth. Journal of the American Dental Association, 81: 101-107.

Okamoto O., Mori O., Morimoto M., Nakao N., and Miyakawa E. (1951) Statistical and genetic studies in hypodontia. The Journal of the Tokyo Dental College Society, 51: 8-10, 39-46, 71-74 (in Japanese).

Osborn R.H., Horowitz S.L., and De George F.V. (1958) Genetic variation in tooth dimension, a twin study of the permanent anterior teeth. American Journal of Human Genetics, 10: 350-355.

Ozaki T. (1960) The reduction index of the Japanese teeth. Acta anatomica Nipponica, 35: 563-577 (in Japanese with English summary).

Peters H., Neubuser A., Kratochwil K., and Balling R. (1998) Pax9-deficient mice lack pharyngeal pouch derivatives and teeth and exhibit craniofacial and limb abnormalities. Genes \& Development, 12: 2735-2747.

Ringqvist M. and Thilander B. (1969) The frequency of hypodontia in an orthodontic material. Svensk Tandläkare Tidskrift, 62: $1535-1541$.

Rose J.S. (1966) Survey of congenitally missing teeth, excluding third molars in 6000 orthodontic patients. Dental Practice, 17: 107-114.

Sakai T. (1957) The correlation between the tooth and the dental arch, the palate and the face in the Japanese. Anthropological Reports, 18: 287-302 (in Japanese with English summary).

SAS Institute (2000) One-Way Layout: The Fit Y by X Pattern, Chapter 4 in JMP Statistics and Graphics Guide JMP Version 4, SAS Institute Inc., Gary, NC, pp. 83-120.

Satokata I. and Maas R. (1994) Msx1 deficient mice exhibit cleft palate and abnormalities of craniofacial and tooth development. Nature Genetics, 6: 348-356.

Shapiro B.L. (1975) Amplified developmental instability in Down's syndrome. Annals of Human Genetics, 38: 429-437.

Sofaer J.A. (1973) A model relating developmental interaction and differential evolution reduction of tooth size. Evolution, 27: $427-434$.

Sofaer J.A., Chung C.S., Niswander J.D., and Runck D.W. (1971a) Developmental interaction, size and agenesis among permanent maxillary incisors. Human Biology, 43: 36-45.

Sofaer J.A., Bailit H.L., and MacLean C.J. (1971b) A developmental basis for differential tooth reduction during hominid evolution. Evolution, 25: 509-517. 
Terasaki T. and Siota K. (1954) Hypodontia. Journal of the Japanese Stomatological Society, 3: 88-93 (in Japanese).

Thompson G.W. and Popovich F. (1974) Probability of congenitally missing teeth: results in 1,191 children in the Burlington Growth Centre in Toronto. Community Dentistry and Oral Epidemiology, 2: 26-32.

Townsend G.C. (1983a) Tooth size in children and young adults with trisomy 21 (Down) syndrome. Archives of Oral Biology, 28: $159-166$.

Townsend G.C. (1983b) Fluctuating dental asymmetry in Down's syndrome. Australian Dental Journal, 28: 39-44.

Tukey J. (1953) A problem of multiple comparisons. Manuscript, Princeton University, NJ.

Yamada H. (1977) Factor analysis of human teeth, dental arch and palate. The Aichi-Gakuin Journal of Dental Science, 15: 267287 (in Japanese with English summary).

Yamada H. and Hanamura H. (1993) The relationship of congenitally missing third molar to other missing teeth in human den- tition. Japanese Journal of Oral Biology, 35: 197-204 (in Japanese with English summary).

Yamada H., Kondo S., and Hanamura H. (2005) Tooth size and molar crown characters of individuals with third molar agenesis in Japanese. Anthropological Science (Japanese Series), 113: 109-117 (in Japanese with English summary).

Yamazaki H. (1960) Three families with hypodontia. Journal of the Japanese Stomatological Society, 27: 120-131 (in Japanese).

Volk A. (1963) Über die Häufigkeit des Vorkommens von fehlenden Zahnanlagen. Schweizerische Monatsschrschr Zahnheilkd, 73: 320 .

Werther R. and Rothenberg F. (1939) Anodontia; a review of its etiology with presentation of case. American Journal of Orthodontics, 25: 61-81.

Zimmerman S. (1967) Congenitally missing teeth. Journal of the American Dental Association, 74: 298-299. 